\title{
Design, Realization and Experiments with a new RF Head Probe Coil for Human Vocal Tract Imaging in an NMR device
}

\author{
J. Přibil, D. Gogola, T. Dermek, I. Frollo \\ Institute of Measurement Science, SAS, Dúbravská cesta 9, SK-841 04 Bratislava, Slovakia, umerprib@savba.sk
}

\begin{abstract}
Magnetic resonance imaging (MRI) is nowadays widely used in medicine for diagnostic imaging and in research studies. The modeling of the human vocal tract acoustics has recently attracted considerable interest. This paper describes the design, realization and first MR scan experiments with a new head probe coil for vocal tract imaging in the open-air MRI equipment working in a weak magnetic field up to $0.2 \mathrm{~T}$. The paper also describes an experimental setting for sound recording during the MR imaging.
\end{abstract}

Keywords: NMR imaging, field calculation, RF probe coil, vocal tract modeling

\section{INTRODUCTION}

$\mathrm{T}$ HE NON-INVASIVE magnetic resonance (MR) scanning of the human vocal tract volume enables to develop the three-dimensional (3D) computer models of the vocal tract. These 3D models are necessary for understanding the basic physical principles for the creation of human speech and voice as close to reality as possible. The primary volume models of the human acoustic supraglottal spaces created from the MR images can then be transformed into the 3D finite element (FE) models [1]. Such models are helpful for modeling the real clinical situation, such as influence of various inborn defects in human supraglottal spaces on speech and voice or simulations of various postsurgical states in patients [2]. The quality of the developed FE models has to be checked by a sufficiently accurate numerical simulation of the subject phonation during the NMR scanning and therefore the simultaneous acoustic recording of subject voice during the scan procedure is very important [3], [4].

Head probe coils are commonly produced for the magnetic resonance imaginer (MRI) equipment working with strong magnetic field, but these systems produce a lot of unwanted acoustic noise. The solutions to the acoustic noise problems are low field MR scanners, but these are not usually provided with the head/neck coils. Therefore, this study focused on the development of a new MR receiving head coil, for a tomograph with low magnetic field to be used primarily in human vocal tract imaging.

\section{SUBJECT \& METHODS}

\subsection{Theoretical calculation and design of the head coil}

For the best signal to noise ratio, a radio frequency (RF) receiving coil should be exactly matched to the preamplifier and tuned to the main working frequency of MRI devices. For this reason, variable capacitors must be used [5]. Because these capacitors are part of the resonance circuit, they should be situated near a receiving coil, as close as possible. This resonance circuit is placed into the basic magnetic field $B_{0}$ of the MRI device; therefore variable capacitors with ultra-low magnetic susceptibility must be used. Next requirements for capacitor MR probe include high quality probe (with minimal losses), high voltage immunity, ultra-low piezoelectric effects, and high temperature stability [6]. During sample excitation by the radiofrequency (RF) pulse, the coil can induce a high voltage. For this reason, the detuning circuit was used as a part of the resonance circuit of coil, like a protection of the preamplifier from this high voltage. For a good homogeneity in the center, the probe coil was designed as a circular solenoid see Fig.1.

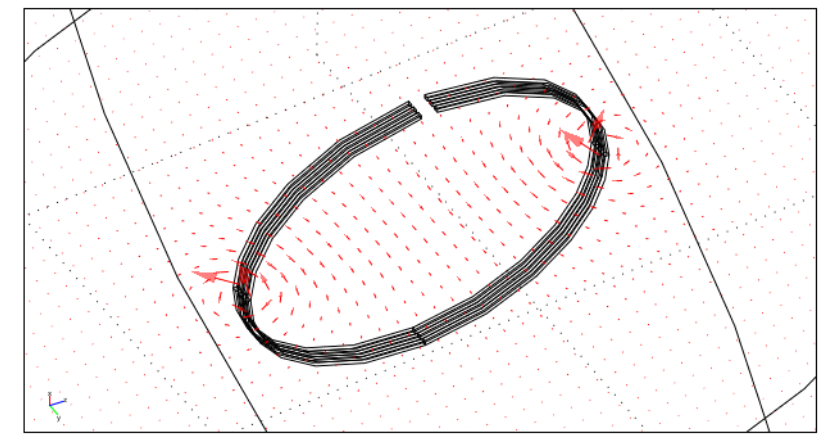

a)

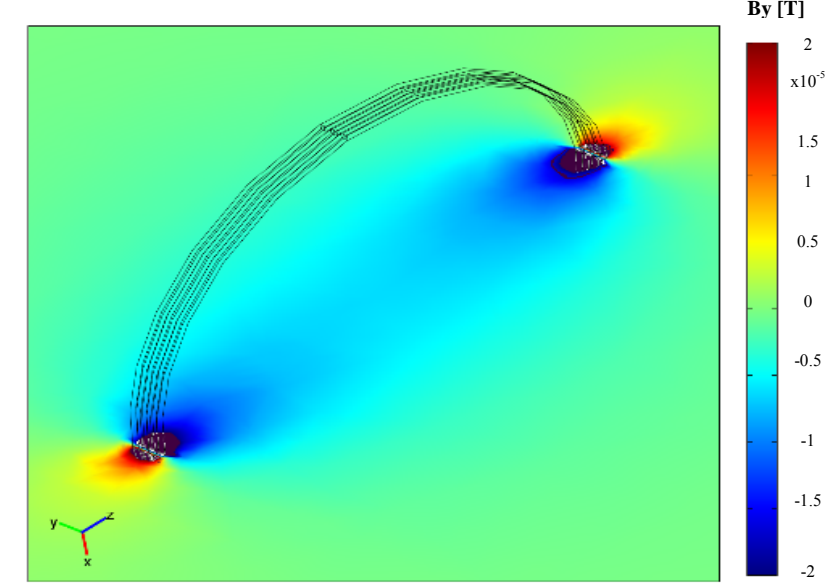

b)

Fig.1. Simulation results of the developed probe coil: vector map of a magnetic field (a), the $y$-component of a magnetic flux density in $[\mathrm{T}](\mathrm{b})$. 
Signal to noise ratio (SNR) and contrast to noise ratio (CNR) were calculated from the image which was produced with our coil. The magnetic field $B_{z}$ of a simple circular coil can be calculated as described in [6]

$$
B z=\frac{\text { ElipticK }[k]+\frac{\text { ElipticE }[k]\left(R_{1}^{2}-r^{2}-\left(z-z_{1}\right)^{2}\right)}{\left(R_{1}-r\right)^{2}+\left(z-z_{1}\right)^{2}}}{\sqrt{\left(z-z_{1}\right)^{2}+\left(R_{1}+r\right)^{2}}},
$$

where

$$
k=\frac{4 R_{1} r}{\left(R_{1}+r\right)^{2}+\left(z-z_{1}\right)^{2}},
$$

and functions ElipticK, ElipticE represent the complete elliptic integral of the first or the second kind, respectively [7]. This holds for $R_{1}=0.09$ [m], as a radius of the coil, $r$ denotes a variable in radius direction, $z$ is variable in $z$ direction, $z_{1}=0.007[\mathrm{~m}]$ is distance of each wire loop. The induction of magnetic field $B_{1}$ in the center of the solenoid coil is described by the following equation [8]

$$
B_{1}=\frac{\mu_{0} n I}{2 h \sqrt{1+4\left(\frac{r}{h}\right)^{2}}},
$$

where $\mu_{0}$ is permeability of free space, $n$ is number of turns, $I$ is electric current, $h$ is height of the coil, and $r$ is radius of the coil.

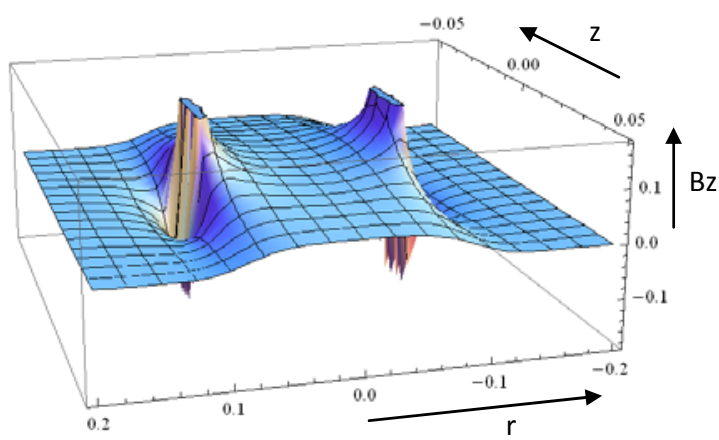

a)

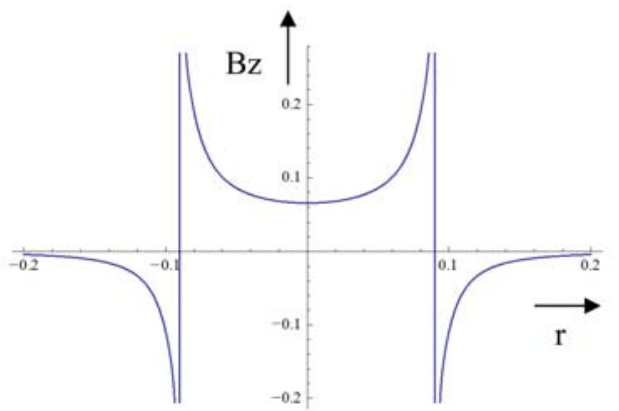

b)

Fig.2. Image of the magnetic field induction: in a 3D space (a), and in a plane with $\mathrm{z}=0(\mathrm{~b})$.
For the RF coil design, simulation and calculation, different types of SW tools were used. Homogeneity of the magnetic field of coil was calculated from the simulation in the program Comsol Multiphysics, theoretic calculation of magnetic induction (see Fig.2) was made with the help of the Mathematica program.

\subsection{Practical realization of the head coil}

The designed head coil was developed and practically realized for using in open-air MR imager $0.178 \mathrm{~T}$ Esaote Escan OPERA [9]. This coil consists of three turns of wire with diameter $2.5 \mathrm{~mm}$. Loop diameter is $22 \mathrm{~cm}$. Each thread is divided into two parts which are connected by the capacitors, as shown in an equivalent circuit in Fig.3.

Detuning circuit was implemented into the receiving coil, for improved attenuation. This detuning circuit is active when the receiving signal reaches or is higher than $1 \mathrm{~V}$. A discrete pre-amplifier is powered by an external $12 \mathrm{~V}$ battery due to minimizing the additive noise of the received RF signal - see block diagram in Fig.4.

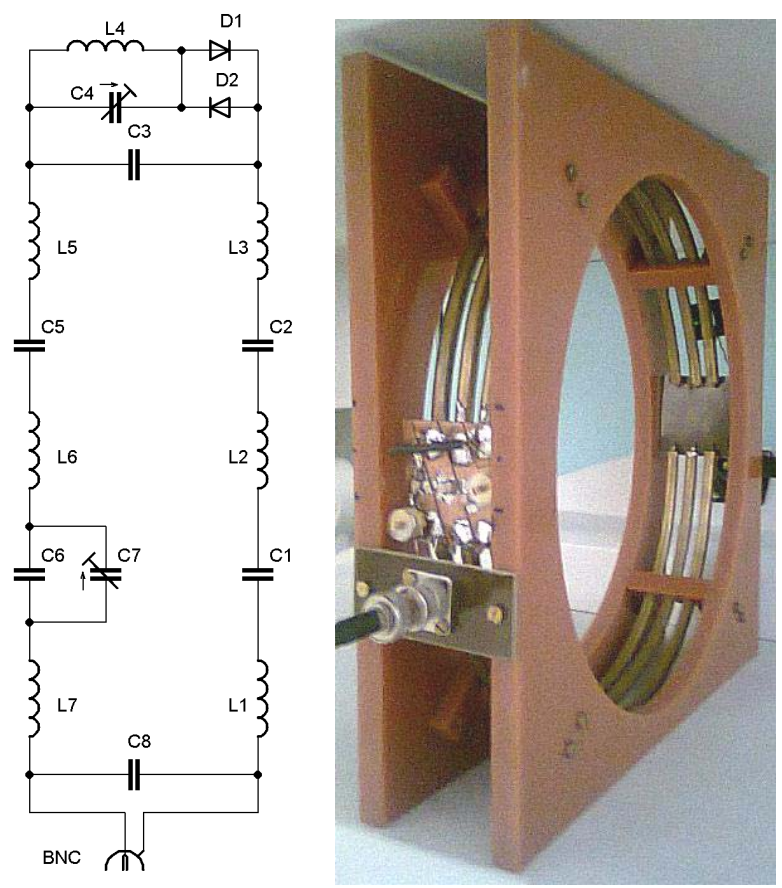

Fig.3. Equivalent circuit of RF head probe coil (left), photo of first realization of the probe (right).

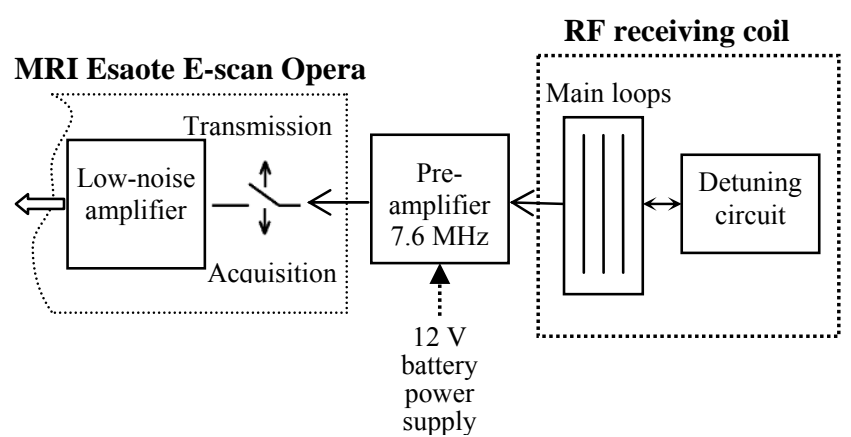

Fig.4. Block diagram of the connection of the receiving coil into the ESAOTE system and illustration of the signal path. 
Receiving coil in our MRI device is oriented perpendicular to the vector of a radiofrequency field. The reason is to minimize the received RF signal during transmission, while the transmission RF coil is located on the back of the scan area as can be seen on the photo in Fig.5. The Esaote software allows manual settings for coil recognition after connection to the MRI device [9]. That's the reason, why hardware coil recognition is not necessary and a special resistance terminator is not necessary to be implemented in a new RF coil.

\section{COMPARISON OF DIFFERENT MR SCAN SEQUENCES AND PARAMETER SETTINGS FOR VOCAL TRACT IMAGINING}

During the MRI session, the assumed physical position of the tested subject is lying on the back with the neck placed inside the RF coil and between two gradient coils as demonstrated in a photo in Fig.5. The obtained image of our first experiments with developed head probe coil is depicted in Fig.6. Resulting SNR of the tissues surrounding the vocal tract in the measured image were as follows: soft palate 26.84, tongue 22.36 and epithelium 15.36. The CNR of the tissues against the cavities was as follows: soft palate 26.45 , tongue 14.96 and epithelium 21.96.

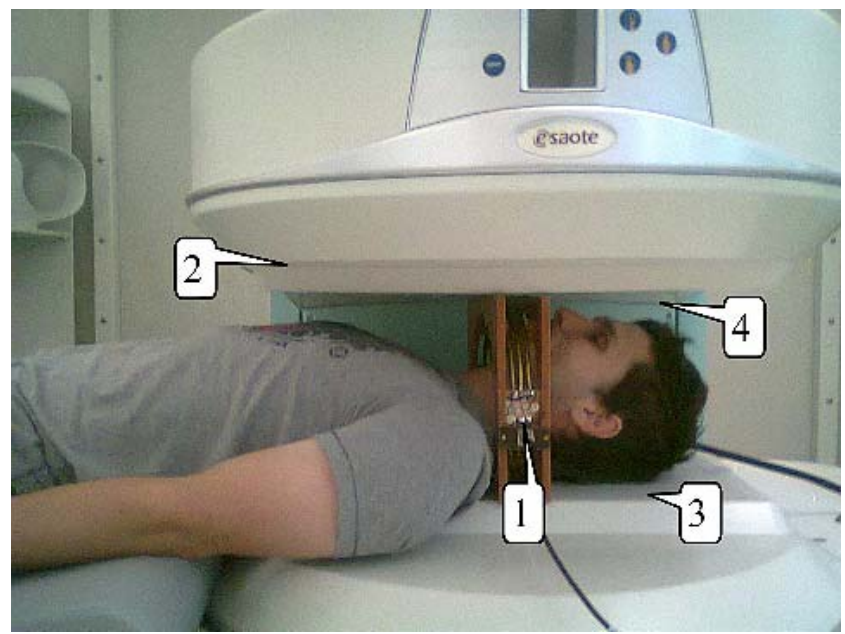

Fig.5. Photo of a male testing person in MRI Opera during scan of the vocal tract: (1) - the developed head probe coil, (2) - the upper gradient coil and the permanent magnet, (3) - the lower gradient coil and the permanent magnet, (4) - the transmission coil.

Due to the low basic magnetic field $B_{0}$ (up to $0.2 \mathrm{~T}$ ) of our MRI machine, the scanning times are longer than those in the MR tomographs working with the 1.5 or $3 \mathrm{~T}$ superconductivity magnet. Chosen type of the sequence together with basic scan parameters - repetition time (TR) and echo time (TE) - has significant influence on the scanning time. Values of these parameters primarily follow from the chosen type of the scanning sequence. They can be also manually slightly changed, but their final values depend on the settings of other scan parameters - field of view (FOV), number of slices, slice thickness, etc. Five types of scanning sequences are implemented in the MRI device Escan OPERA [8], but not all of this is usable for the vocal tract imagining. As follows from our previous experiments, only the $3 \mathrm{D}$ and Hi Res sequences can bring pictures in sufficient resolution and quality. Before starting the scan sequence, the quality factor (QF) parameter is automatically pre-calculated to obtain a picture of sufficient quality. Typical scanning time is between 1 and 2 minutes, although for the 3D or Hi Res scan sequences, more than 2 minutes are necessary - see complete MR image with all setting of scan parameters included in Fig.7.

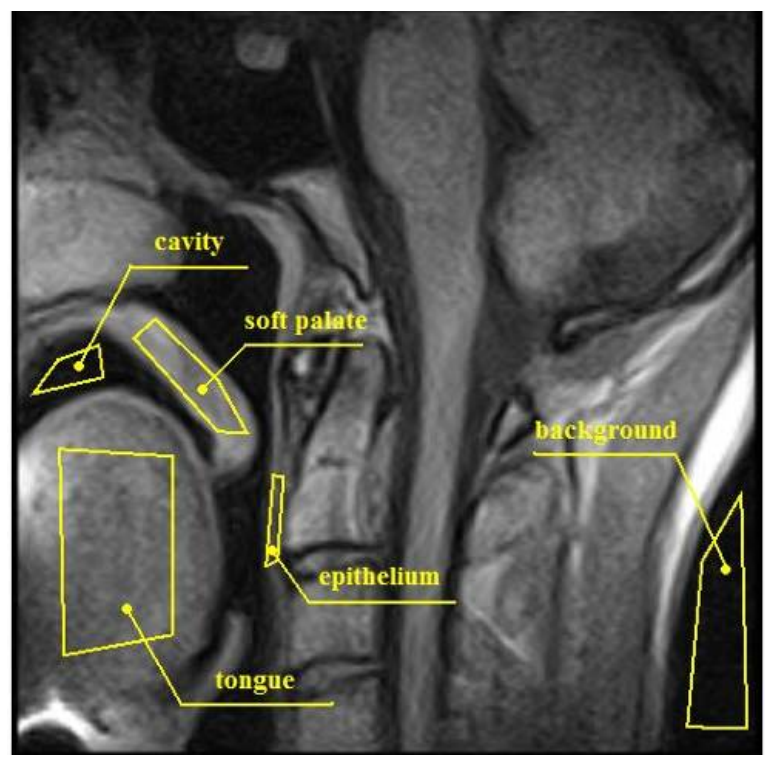

Fig.6. Detailed MR image of the vocal tract using the SE T2 scan sequence (with chosen parameters $\mathrm{TR}=900 \mathrm{~ms}$, $\mathrm{TE}=26 \mathrm{~ms}$, slice thickness $=5 \mathrm{~mm}$ ), including described regions of interest (ROI).

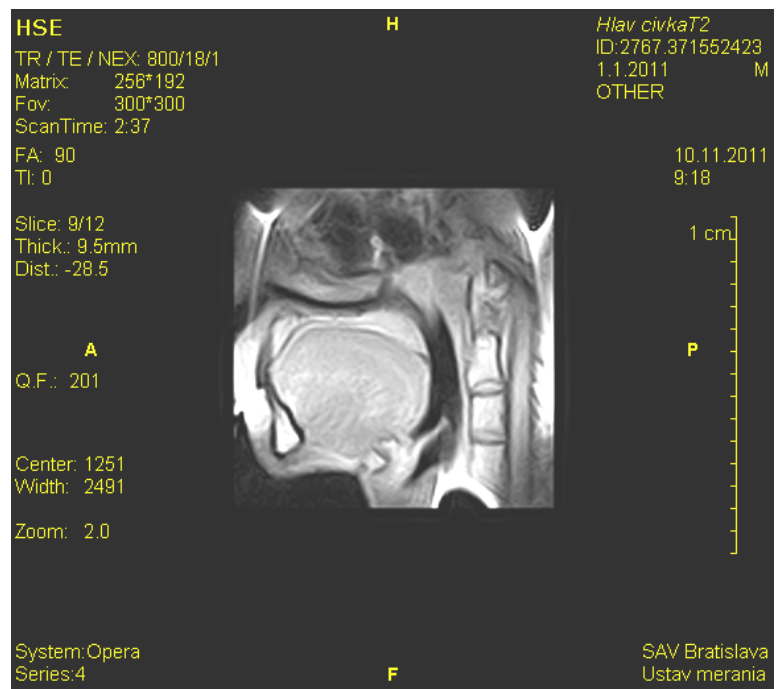

Fig.7. Obtained complete MR image of the male vocal tract in a sagittal plane including the current setting of scan parameters (when using the Hi Res Spin Echo scan sequence).

Predicted picture QF and the scan time were considered as the main criteria for the scan sequence type and choice of parameter settings. Therefore a detailed analysis and comparison for two types of scan sequences were realized. Obtained results are presented in numerical form (see values stored in Tables 1-2) complemented by a comparison of MR images in Fig.8-9. 

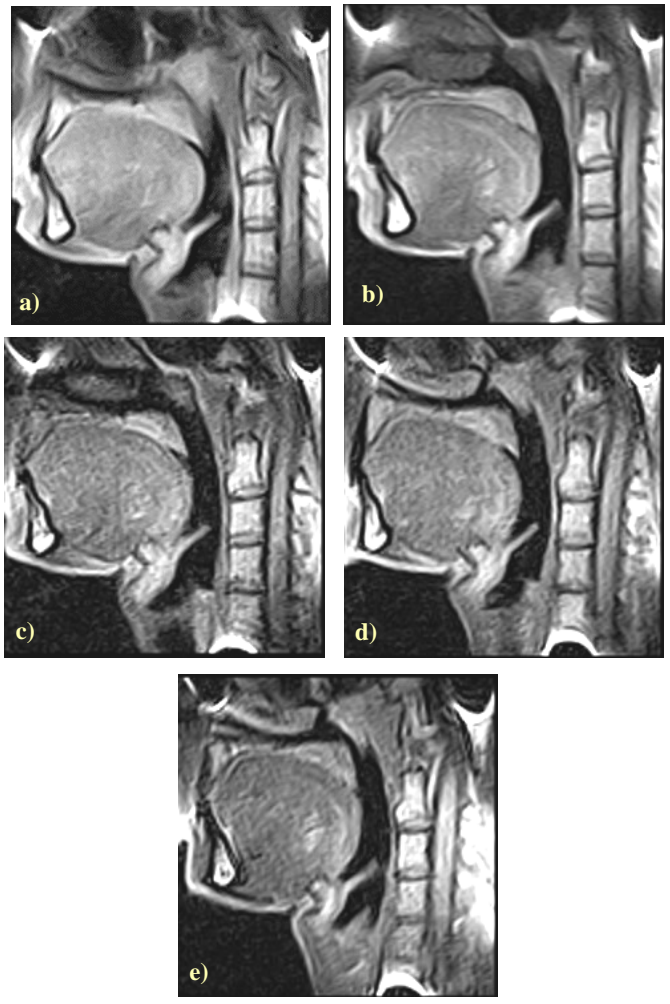

Fig.8. Set of MR images of the male vocal tract in a sagittal plane using the Hi Res SE scan sequence documenting a dependence of used scan parameters corresponding to values of Table 1 .
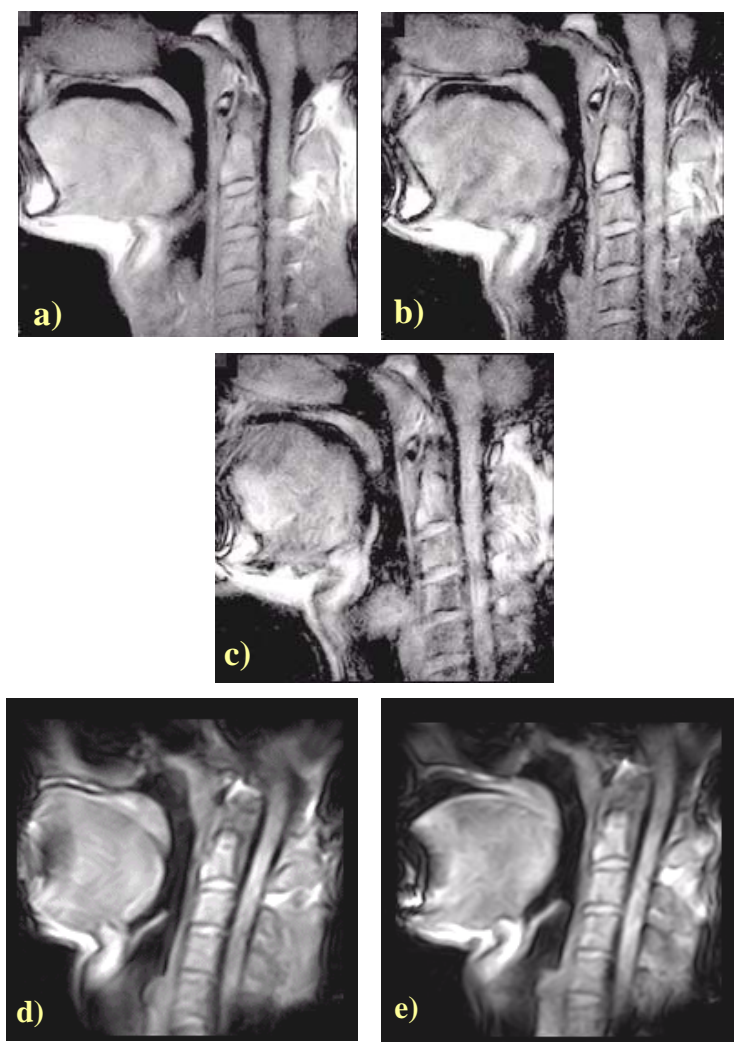

Fig.9. Set of MR images of the male vocal tract in a sagittal plane using the 3D SSF scan sequence documenting a dependence of used scan parameters corresponding to values of Table 2 .
Table 1. Details of the analyzed scanning sequences (Hi Res SE type, $\mathrm{TE}=26 \mathrm{~ms}$ ) with settings of 10 slices, FOV $300 \times 300 \times 192$.

\begin{tabular}{|c|c|c|c|c|c|}
\hline Case & $\begin{array}{c}\text { TR } \\
{[\mathrm{ms}]}\end{array}$ & $\begin{array}{l}\text { Matrix } \\
\text { size }\end{array}$ & $\begin{array}{l}\text { Thick } \\
\text { [mm] }\end{array}$ & $\begin{array}{c}T_{\mathrm{SCAN}} \\
{[\mathrm{min}: \mathrm{sec}]}\end{array}$ & $\begin{array}{l}\text { QF } \\
{[-]}\end{array}$ \\
\hline a) & 800 & $256 \times 192$ & 9.5 & $1: 33$ & 222 \\
\hline b) & 500 & $256 \times 192$ & 7.5 & $1: 39$ & 196 \\
\hline c) & 800 & $256 \times 192$ & 4.5 & $1: 33$ & 100 \\
\hline d) & 800 & $192 \times 192$ & 4.5 & $1: 33$ & 134 \\
\hline e) ${ }^{*}$ & 600 & $192 \times 192$ & 3.5 & $2: 10$ & 122 \\
\hline
\end{tabular}

Table 2. Detail of analyzed scanned sequences (3D SSF type, $\mathrm{TE}=10 \mathrm{~ms}, 1$ accumulation).

\begin{tabular}{cccccc}
\hline Case & $\begin{array}{c}\text { TR } \\
{[\mathbf{m s}]}\end{array}$ & Matrix size & $\begin{array}{c}\text { Thick } \\
{[\mathbf{m m}]}\end{array}$ & $\begin{array}{c}\boldsymbol{T}_{\text {SCAN }} \\
{[\mathbf{m i n}: \mathbf{s e c}]}\end{array}$ & $\begin{array}{c}\text { QF } \\
{[-]}\end{array}$ \\
\hline a) & $40^{\mathbf{1 )}}$ & $200 \times 200 \times 34$ & 10.2 & $2: 32$ & 227 \\
b) & $40^{1)}$ & $200 \times 200 \times 22$ & 9.4 & $2: 56$ & 201 \\
c) & $40^{1)}$ & $200 \times 200 \times 22$ & 4.7 & $2: 56$ & 128 \\
d) & $30^{2)}$ & $256 \times 200 \times 16$ & 7.5 & $1: 56$ & 335 \\
e) & $30^{2)}$ & $256 \times 200 \times 16$ & 9.4 & $1: 36$ & 398 \\
\hline \multicolumn{5}{c}{ 1) $\mathrm{FOV}=200 \times 200 \times 130,{ }^{2)} \mathrm{FOV}=400 \times 400 \times 150$}
\end{tabular}

\section{EXPERIMENTS WITH PHONATION DURING THE MR SCAN}

To obtain MR pictures with sufficient quality without any artifacts, interaction with the stationary magnetic field $B_{0}$ in the MRI scanning area must be eliminated during speech signal pick up. The homogeneity of low-magnetic field $B_{0}$ into the scan area of the MRI machine can be mapped by special phantoms [10]. Therefore, we use an arrangement, where the pick-up microphone is located in a sufficient distance from the noise signal source outside the magnetic field area. The bed with the testing person was set to 150 deg. position (originated from right corner opposite the temperature stabilizer device - see Fig.10), the speech pickup microphone was placed at the $30 \mathrm{deg}$. position in the distance of $50 \mathrm{~cm}$ from the head coil. The head of the testing person was inserted into the knee coil (see also item 1 in Fig.5), the background noise level (generated mainly by a temperature stabilizer) was $I_{0}=55 \mathrm{~dB}$ [11].

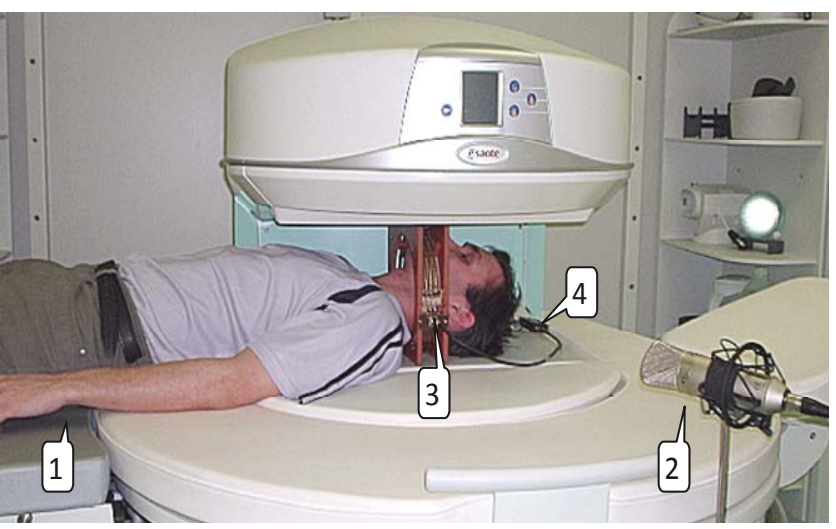

Fig.10. A speech recording experiment arrangement: (1) - bed with testing person, (2) - the pickup microphone, (3) - head RF coil, (4) - external RF pre-amplifier. 


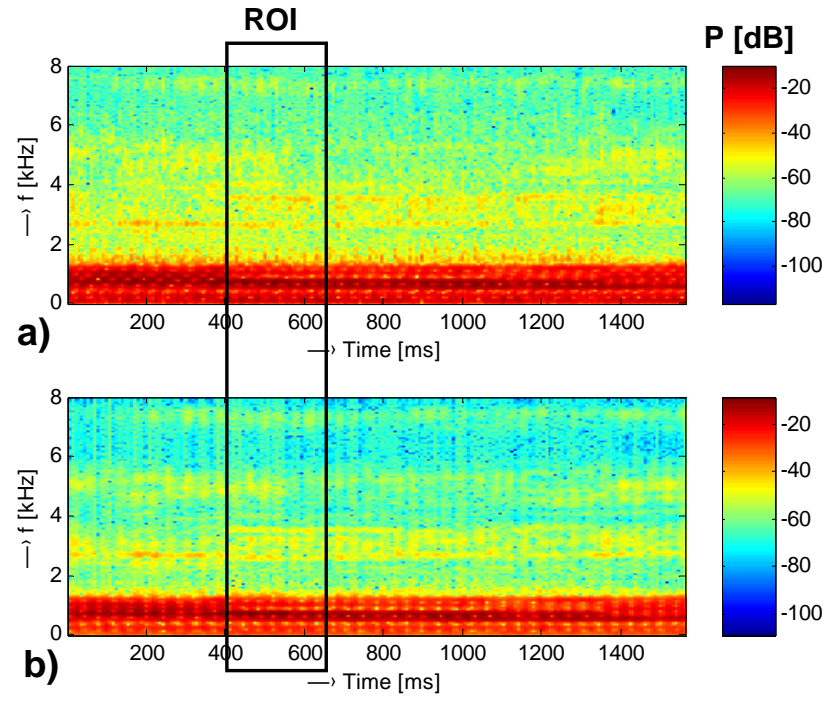

Fig.11. Spectrograms of the processed long vowel "a" (male voice, $\mathrm{F} 0 \approx 130 \mathrm{~Hz}, f_{\mathrm{s}}=16 \mathrm{kHz}$,) recorded during the MR scan using the $3 \mathrm{D}$ SFF sequence: original speech and noise signal (a), reconstructed signal after cepstrum noise reduction (b).

a)
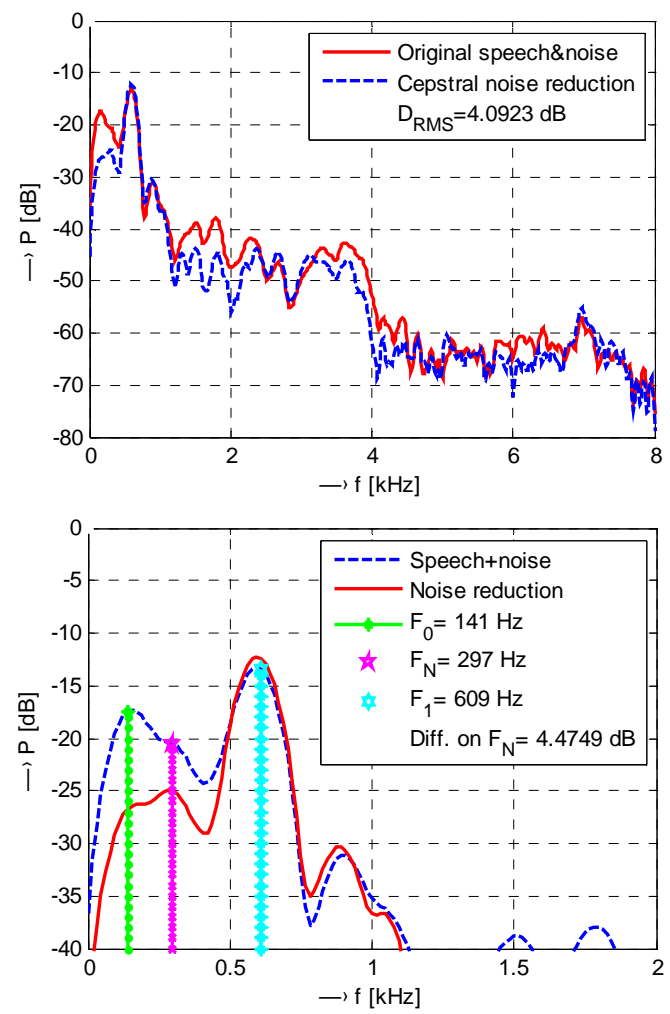

b)

Fig.12. Corresponding mean periodograms of selected $250 \mathrm{~ms}$ time interval (ROI) : summary periodograms for full frequency range up to $f_{\mathrm{s}} / 2$ (a), detailed parts with determined $\mathrm{F} 0, F_{\mathrm{N}}, F_{1}$ positions, and the $\Delta P$ value in $[\mathrm{dB}]$ on the $F_{\mathrm{N}}$ position (b).

The used MRI device has a control console based on the standard personal computer, but due to safety and stability of the system it is not possible to add any external device (using serial, USB, Blue-Tooth, or the Wi-Fi connection). Therefore, the speech signal was recorded with the help of the M-Audio FireWire 1814 equipment connected to a separate personal computer (laptop) and picked up by the professional condenser microphone with the figure-of-eight pickup pattern. The synchronization of the speech recording and the MR scan process was realized manually on the side of a console operator. By reason that the scan times are about 1 minute, the phonation is performed in blocks with mean duration of 8,15 , or 30 seconds depending on physical condition of the tested person.

Recorded speech signal contains also an additional noise that is mainly produced by gradient coils due to rapidly changing Lorentz forces during fast switching inside the environment of the MRI device [12]. This noise has a mechanical character, and can be processed in spectral domain [13] - see spectrograms obtained from phonation of the long vowel "a:" during MR scan in Fig.11. For noise reduction, we used the method based on the cepstral speech model [14]. We applied the limitation and clipping the "peaks" corresponding to the harmonic frequencies of mechanical noise on the real cepstrum [11]. By this approach we obtained the noise reduction up to $8 \mathrm{~dB}$ on the $F_{\mathrm{N}}$ position (the first harmonic frequency of the mechanical noise), which is sufficient for next speech signal processing.

Setting of the basic parameters of the scanning sequences (TR and TE) had significant influence on properties of the acoustic noise finally produced by the MRI device. Values of these parameters primarily follow from the chosen type of the scanning sequence. User setting of the TR parameter affects mostly the $F_{\mathrm{N}}$ values - see an example in Fig.12 of mean periodograms calculated by the Welch method.

\section{DisCUSSION AND CONCLUSION}

The prototype of receiver coil design with partitioning of turns seems to be feasible for obtaining reasonable signal to noise ratio. The calculated contrast to noise ratio of the tissues surrounding the vocal tract against the cavities was acceptable, and thus the vocal tract was easily recognized in the image.

According to the design, a head probe coil was constructed and afterwards tested in E-Scan Opera ESAOTE tomograph. From our results it is clear that our head coil developed for low-field MR scanners is suitable for vocal tract MRI, as seen in Fig.7. Images with relative high SNR (see sets of MR scan obtained by Hi Res and 3D scan sequences in Fig.8 and Fig.9) can be measured with only two averages, thus in a reasonably short acquisition time. Imaging of the throat and mouth cavity in short time along with voice recording was therefore possible without enormous acoustic noise. In our case, the mouth cavity was not fully visible due to the imaging sequence restrictions (applied FOV). The increase of FOV is the focus of our future developments.

As follows from phonation experiments, the noise produced by gradient system of MRI device influences the quality of the recorded speech signal. The documentary Fig.11 also shows determined values of speaker's fundamental frequency $\mathrm{F} 0$ and first formant $F_{1}$ position, in contraindication with the mechanical noise $F_{\mathrm{N}}$ frequency of the chosen ROI signal area. Typical situation occurs when the $F_{\mathrm{N}}$ frequency lies between the speaker's F0 and first formant $F_{1}$ position (see Fig.12b). As we applied the speech signal analysis tools to MRI noise analysis, theses signal's 
peaks were treated as the pitch pulses in the noise and the analysis window length $w_{\mathrm{L}}$ depended on their period. When this period is too long $\left(F_{\mathrm{N}}<6 \mathrm{~Hz}\right)$, the spectral analysis method does not give correct results and we can obtain only inaccurately determined values.

The designed low-field MR probe head coil has fair properties, but not yet optimal. The reasons why it is so for a proper MRI measurement are:

a) a relatively too long capturing time related to phonation capabilities of the subject,

b) too high thickness of the MR slices,

c) not fully visible vocal tract from the vocal folds to the lips due to a small FOV.

The advantage of the system is in the design method of the device noise elimination by especially developed software which enables to measure the acoustic characteristics of the subject during phonation in the NMR device with a good accuracy. Resulting quality of cleaned speech can be evaluated by subjective methods (listening test [15]) or with the help of speech recognition methods using evaluation by hidden Markov models or statistical approaches [16], [17] (the objective ones), as is usual in the speech processing area.

In further research we will primarily focus on the selection of more suitable materials for the construction of the coil to increase the coil quality and thus improve the signal to noise ratio. For the same purpose a high impedance differential amplifier will be the goal of further research. For automatic fine-tuning and better compatibility with E-Scan Opera ESAOTE system the air variable tuning capacitor will be replaced by the varicaps.

\section{ACKNOWLEDGMENT}

The work has been supported by the Grant Agency of the Slovak Academy of Sciences (VEGA 2/0090/11 and 2/0160/10), State program SPVV No. 2003SP200280203), and State project APVV-0513-10.

\section{REFERENCES}

[1] Vampola, T., Horáček, J., Švec, J.G. (2008). FE modeling of human vocal tract acoustic: Part I Production of Czech vowels. Acta Acustica United with Acustica, 94, 433-447.

[2] Vampola, T. et al. (2008). FE modeling of human vocal tract acoustics: Part II - Influence of velopharyngeal insufficiency on phonation of vowels. Acta Acustica United with Acustica, 94, 448-460.

[3] Malinen, J., Palo, P. (2009). Recording speech during MRI: Part II. In Proc. of the $6^{\text {th }}$ Int. Workshop Models and Analysis of Vocal Emissions for Biomedical Applications, December 14-16, 2009. Frenze University Press.
[4] Aalto, D. et al. (2011). Recording speech sound and articulation in MRI. In Proc. of the Int. Conference on Biomedical Electronics and Devices Biodevices 2011. Rome, Italy, 168-173.

[5] Andris, P., Frollo. I. (2007). Varicaps for NMR receiving coil matching and sensitivity changes. Journal of Electrical Engineering, 58 (5), 291-293.

[6] Doty, F.D. (2007). Solid state NMR probe design. In Encyclopedia of Magnetic Resonance. Wiley Online Library, DOI: 10.1002/9780470034590.emrstm1001.

[7] Prasolov, V., Solovyev, Y. (1997). Elliptic Functions and Elliptic Integrals. American Mathematical Society.

[8] Frollo, I. (1989). Generation and application of gradient magnetic fields in NMR measurement and imaging. Scientific Instrumentation, 4 (2), 41-51.

[9] Esaote S.p.A. (2008). E-scan Opera. Image Quality and Sequences Manual. 830023522 Rev. A.

[10] Frollo, I. et al. (2010). Measurement and imaging of planar electromagnetic phantoms based on NMR imaging methods. Measurement Science Review, 10 (3), 97-101.

[11] Přibil, J., Horáček, J., Horák, P. (2011). Two methods of mechanical noise reduction of recorded speech during phonation in an MRI device. Measurement Science Review, 11 (3), 92-98.

[12] Moelker, A., Wielopolski, P.A., Pattynama, M.T. (2003). Relationship between magnetic field strength and magnetic-resonance-related acoustic noise levels. Magnetic Resonance Materials in Physics, Biology and Medicine, 16, 52-55.

[13] Tomasi, D., Ernst, T. (2006). A Simple theory for vibration of MRI gradient coils. Brazilian Journal of Physics, 36, 34-39.

[14] Vích, R., Přibil, J., Smékal, Z. (2001). New cepstral zero-pole vocal tract models for TTS synthesis. In Proc. of the IEEE Region 8 EUROCON'2001, Vol. 2, Section S22-Speech Compression and DSP, 458-462.

[15] Grůber, M., Matoušek, J. (2010). Listening-test-based annotation of communicative functions for expressive speech synthesis. In Text, Speech and Dialogue: Proc. of the $13^{\text {th }}$ Int. Conference TSD 2010, LNCS 6231. Springer, 283-290.

[16] Vich, R., Nouza, J., Vondra, M. (2008). Automatic speech recognition used for intelligibility assessment of text-to-speech systems. In Verbal and Nonverbal Features of Human-Human and Human-Machine Interactions, LNCS 5042. Springer, 136-148.

[17] Srinivasan, S., DeLiang, W. (2010). Robust speech recognition by integrating speech separation and hypothesis testing. Speech Communication, 52, 72-81.

Received February 12, 2012. Accepted May 31, 2012. 